\title{
Experiencia formativa en escuelas monoculturales de la Araucanía, Chile: memoria de mujeres en Cholchol y Maquehue ${ }^{1}$
}

\author{
Formative experience in monocultural schools in Araucanía, \\ Chile: memories of women in Cholchol and Maquehue
}

Experiência de formação em escolas monoculturais em Araucanía,

Chile: memória das mulheres em Cholchol e Maquehue

Xürümtuwün zugu kiñe rume kimün Gulu mapu chijkatuwe mew, Chile: Epu Cholchol ka Makewe zugu ñi tukulpan

\author{
Claudia Huaiquián Billeke ${ }^{2}$ \\ Universidad Católica de Temuco, Chile \\ Juan Guillermo Estay Sepúlveda ${ }^{3}$ \\ Universidad Católica de Temuco, Chile \\ Universidad Adventista de Chile
}

Recepción: 26/03/2019

Evaluación: 15/05/2019

Aceptación: 23/05/2019

Artículo de Investigación

DOI: https://doi.org/10.19053/01227238.9970

\section{RESUMEN}

Esta investigación se enmarca en los resultados preliminares de un proyecto Fondecyt regular. Este artículo tiene como objetivo develar descriptivamente la memoria social de cinco mujeres, abuelas indígenas de la comunidades mapuche de Maquehue y del sector Cholchol, con un rango de edad que oscila entre los sesenta y los setenta años, desde sus subjetividades, sus particularidades y sentimientos; el texto aborda el problema de la invisibilización de la sabiduría ancestral indígena de las abuelas mapuche, las principales agentes de transmisión cultu-

1 Este artículo forma parte del Proyecto Fondecyt regular n. ${ }^{\circ} 1191016$ "Consolidación de la escuela monocultural en territorio mapuche, periodo post-reduccional (1929-1973). Colonialidad republicana e invibilización del mapun kimun cuyo investigador resposable es el Dr. Juan Mansilla Sepúlveda.

2 Doctora en Ciencias de la Educación. Directora del Centro de Evaluación y Estimulación Temprana Universitaria (CEETU-UCT). Universidad Católica de Temuco, Chile. Correo electronico: chuaiquian@uct.cl

3 Profesor de Historia y Geografía. Académico Universidad Católica de Temuco, Chile. Integrante Núcleo Investigación Universidad Adventista de Chile, Chile. Correo electrónico: juanguillermoestay@yahoo.es 
ral de generación en generación. El marco teórico se orienta a partir del enfoque intercultural, y, por otra parte, el modelo formativo mapuche Kimeltuwun, (proceso educativo mapuche) el cual se vehiculiza a través de epew (cuentos), peumas (suenos) y piam (relatos antiguos). Los relatos fueron socializados desde sus propios territorios, donde compartieron sus vivencias escolares, lo cual, indudablemente, enriqueció la experiencia investigativa. La reconstrucción de la memoria surge del recuerdo de la escolarización monocultural que vivieron en su infancia. Los datos se recogieron a través de entrevista en profundidad y nuw tram, al amparo de un acercamiento intercultural al campo. Los resultados dan cuenta de cómo se fueron invisibilizando sus conocimientos ancestrales en la educación que recibieron, cargada de disciplina y rigidez, centrada siempre en roles domésticos (lavar platos, limpiar pisos, buscar leña), alejados completamente de su propio reconocimiento, su identidad, su autosuperación y su participación social, sin considerar su legado ancestral y todo su contexto. La escuela invade su cultura, la ignora, alejándose de su esencia mapuche, que no es otra que la vida en armonía con la naturaleza.

Palabras clave: mujeres mapuche; memoria social; sentimiento de subordinación.

\section{ABSTRACT}

This research is framed in the preliminary results of a regular Fondecyt project and its aim is to reveal and describe the social memory of five women who are indigenous grandmothers of the Mapuche communities in Maquehue and Cholchol areas. All of them aged between sixty and seventy years. Based on subjectivities, particularities, and feelings of the Mapuche grandmothers, main agents of intergenerational cultural transmission, the text addresses the problem of the invisibility of indigenous ancestral wisdom. The theoretical framework is based on the intercultural approach and, on the Mapuche formative model Kimeltuwun (Mapuche educational process) which is conveyed through epew (stories), peumas (dreams) and piam (ancient narratives). The stories were socialized in their own territories, just where they shared their school experiences; this factor, undoub- tedly, enriched the research experience. The reconstruction of memory arises from the monocultural schooling they shared in their childhood. The data were collected through in-depth interviews and nuw tram, as part of an intercultural approach to the countryside. The results show how their ancestral knowledge became invisible in the education they received, overloaded with discipline and rigidity, and always focused on domestic roles (washing dishes, cleaning floors, looking for firewood), completely distant from their own recognition, identity, self-improvement and social participation, their ancestral legacy and their context was left aside. The school invades its culture, ignores it, moves them away from their Mapuche essence, which consists essentially of a life in harmony with nature.

Keywords: Mapuche women; social memory; feeling of subordination.

\section{RESUMO}

Esta pesquisa é parte dos resultados preliminares de um projeto regular da Fondecyt. Este artigo tem como objetivo descrever descritivamente a memória social de cinco mulheres, avós indígenas das comunidades Mapuche de Maquehue e do setor Cholchol, com uma faixa etária que varia entre sessenta e setenta anos, a partir de suas subjetividades, suas particularidades e sentimentos. ; O texto aborda o problema da invisibilidade da sabedoria ancestral indígena das avós Mapuche, principais agentes de transmissão cultural de geração para geração. 
O referencial teórico é orientado a partir da abordagem intercultural e, de outro, o modelo formativo Kimeltuwun Mapuche (processo educacional mapuche) veiculado através de epew (histórias), peumas (sonhos) e piam (realtaos antigos). . As histórias foram socializadas a partir de seus próprios territórios, onde compartilharam suas experiências escolares, o que, sem dúvida, enriqueceu a experiência de pesquisa. A reconstrução da memória nasce da memória da escolaridade monocultural que eles viveram na infância. Os dados foram coletados através de entrevista em profundidade e nuw tram, sob uma abordagem intercul- tural do campo. Os resultados mostram como o conhecimento ancestral tornou-se invisível na educação que recebiam, cheio de disciplina e rigidez, sempre focado em papéis domésticos (lavar louça, limpar pisos, procurar por lenha), completamente retirado de seu próprio reconhecimento, sua identidade, seu autodesenvolvimento e participação social, sem considerar seu legado ancestral e todo o seu contexto. A escola invade sua cultura, a ignora, afastando-se de sua essência mapuche, que não é outro senão a vida em harmonia com a natureza.

Palavras-chave: Mulheres mapuches; memória social; sentimento de subordinação.

\section{PIKÜNOGETUN}

Tüfa chi inazuam zugu koneltuley ta wünechi xipan zugu kiñe proyecto Fondecyt mew. Tüfa chi chijka zugu zewmagey ta ñi küme pegealgeal kuyfike tukulpazugun nielu ta kechu zomo, kusheke papay tuwlu ta lof Makewe ka tuwlu ta Cholchol mapu püle, nieygün kayuke mari reqleke mari xipantu egün, kizu ñi chum peken mogen egün, kizu ñi chumgen egün ka kizu ñi uamüm egün; faw güxamyegey chijka mew ñi pegelgekenon ta kuyfike kimün nielu ta pu kusheke mapuche papay, tüfa egün je ta rulpakimünniekelu fijke choyün che mew. Chijka kimün koneltuley ta enfoque intercultural ñi zugu mew, ka famgechi ta mapuche ñi kimeltuwün mew, fey tüfey ta yegey epew mew, pewma mew ka piam mew. Tüfeyke güxam nentugey ta kizu ñi pu mapu mew egün, chew ta güxamyeygün ñi chijkatun chijkatuwe ruka mew, fey mew je ta küme newenkoni tüfa chi inazuam küzaw. Fey tüfey chi wüñonentutun zugu nielu ñi logko mew egün xipay ta ñi kuyfike tukulpazugun mew ñi chumgechi chijkatulelgeygün kiñe kimün mew müten püchikalu egün. Gümitugey tüfa chi zugu ramtukan zugu mew ka güxam mew zenkütuwün ta kiñe füluwün intercultural mew. Wenuntuzugu mew kay ta pewfaluwi chumgechi penchulerupun ñi kuyfike kimün jowün mew ta wigka kimün, kümeyawün müten ka re mügeltun mew, re ruka küzaw mew (kücharalin, liftun wejin, kintumamüjün), püntüxipaley kizu ñi mogen mew, ñi chegen mew, ñi chegerupual ka ñi koneltuzugual ñi xokiñche mew, tukulpalay ñi elgerupun kimün chew ñi mülemun. Chijkatuwe ruka wentekoniy ñi mapuche az mogen mew, ijamfi, femgechi püntüxipay ñi mapuchegen, fey tüfey ta küme mogeleal je ixofij mogen egün mülelu mapu mew.

Zichul zugun: pu mapuche zomo; xokiñche ñi tukulpazugun; sentimiento de subordinación

\section{INTRODUCCIÓN}

Este tema es de gran relevancia debido a la interculturalidad presente en la región de la Araucanía. En Chile, el 11,11 \% de la población pertenece a alguno 
de los nueve pueblos indígenas existentes, de los cuales el 84,11\%, corresponde al pueblo mapuche. A nivel local, en la región de la Araucanía el porcentaje de mapuches equivale al $31,58 \%$ de la población ${ }^{4}$.

En cuanto a la comunidad mapuche, es fundamental considerar la identidad étnica regida por patrones culturales y actitudinales basados en las creencias transgeneracionales. Si bien dichas pautas han sobrevivido a los cambios interculturales en la sociedad chilena, estas se encuentran sometidas a un constante proceso de reactualización, redefiniendo sus costumbres en la permanente incorporación de la visión chilena en las prácticas de crianza y educación ${ }^{5}$.

En términos de respetar la valoración cultural de los pueblos originarios, el organismo encargado de promover, coordinar y ejecutar acciones del Estado chileno en pos de los pueblos indígenas en áreas económicas, sociales y culturales es el CONADI'; sin embargo, si bien lo que se pretende es rescatar y fortalecer la riqueza cultural de Chile, muchas de las acciones que utiliza el Estado generan poca afinidad por parte de los pueblos indígenas.

En necesario mencionar que se considera a la cultura ${ }^{7}$ mapuche como una cultura de oralidad, donde el consejo, conocido como ggülam $^{8}$ (consejo entregado por personas sabias) a través del nütram (conversación) conforma la base del aprendizaje significativo y el traspaso de los conocimientos dentro de la cotidianidad del lof che (comunidad) mediante el acompañamiento y adiestramiento en las prácticas aprendidas gracias a la imitación y el modelado de adultos considerados como referentes en dicha comunidad. De este modo, küga (la familia) adquiere un rol preponderante en la formación de la identidad como mapuche en la formación del che (persona), en el traspaso del kümun (los saberes) y el aprendizaje de las tradiciones familiares y culturales entregadas mediante relatos como los epew (cuentos) y pewmas (sueños) por los kimches (ancianos), que son considerados como sabios referentes de los saberes y el traspaso transgeneracional del kümun .

La imagen del padre se relaciona con el rol de proveedor económico y su dedicación al trabajo agrícola y ganadero. A nivel familiar, se destaca como figura de autoridad y liderazgo en la toma de decisiones, resolución de conflictos, traspaso de conocimiento a los hijos mediante el nütram (conversación), promoviendo la participación activa en la comunidad al grupo familiar ${ }^{10}$.

4 INE, Instituto Nacional de Estadísticas de Chile, “Censo 2012, síntesis de resultados” (Santiago: INE, 2012).

5 Michelle Sadler y Alexandra Obach, Pautas de crianza mapuche. Estudio "Significaciones, actitudes y prácticas de familias mapuches en relación a la crianza y cuidado infantil de los niños y niñas desde la gestación hasta los cinco años" (Santiago: Ministerio de Planificación, 2006).

6 Corporación Nacional de Desarrollo Indígena (CONADI), Ley indígena (Ley n. ${ }^{\circ} 19.253$ d. of. 5..$^{\circ} 10{ }^{\circ}$ 1993) (Temuco: CONADI, 2013).

7 Terry Eagleton, La idea de cultura. Una mirada política sobre los conflictos culturales (Barcelona: Paidós, 2001).

8 Aracely Caro y Julio Terencán, "El Ngülam en el discurso intrafamiliar mapuche", Iberóforum. Revista de Ciencias Sociales de la Universidad Iberoamericana, vol. 1, no. 1 (2006).

9 Christine Gleisner y Sara Montt, Mapuche. Serie introducción histórica y relatos de los pueblos originarios de Chile (Santiago: FUCOA-Fondart, 2014).

10 Caro y Terencán, "El Ngülam". 
Si bien la madre es considerada como la principal educadora en la vida de los hijos, es en la primera infancia donde adquiere un rol fundamental asociado a la resolución de conflictos de faltas leves y la enseñanza de quehaceres domésticos, fomentando la autonomía ${ }^{11}$. A su vez, se reconoce como una figura fundamental en la demostración de afectos y la expresión de emociones y respeto a los demás integrantes de la familia, puesto que en el acompañamiento cotidiano en la formación de los hijos incorpora el uso del ngloon (consejo) en diferentes instancias del nütram (conversación) ${ }^{12}$.

Existen elementos asociados y transmitidos en la cultura ${ }^{13}$, es decir, que la mujer adquiere conocimiento sobre las prácticas del quehacer doméstico y artesanal, y el varón, conocimiento respecto a la vida de campo, principalmente en la ganadería y la agricultura, acompañando al padre en las prácticas cotidianas. Si bien existe una tendencia a fortalecer dichos roles de género ${ }^{14}$, se enfoca en el desarrollo de la autonomía y el funcionamiento doméstico en igualdad de condiciones, la valoración del respeto a los integrantes del grupo familiar y la comunidad, junto con la participación activa de ceremonias y ritos con énfasis en el aprendizaje de pautas culturales y la identificación con un rol específico en las mismas ${ }^{15}$.

Por otro lado, el acceso a la educación formal adquiere gran relevancia al ser considerado una herramienta para la superación de la pobreza en la búsqueda de igualdad de oportunidades en el mundo occidental, desde una mirada intercultural, siendo el desafío de los padres el mantener en la práctica la enseñanza de valores en el traspaso de conocimientos de las tradiciones familiares y culturales ${ }^{16}$.

En el grupo familiar, la figura del anciano ocupa un lugar privilegiado ya que son considerados kimches (sabios) sobre el funcionamiento y la comprensión de la cosmovisión del mundo mapuche, siendo el rol fundamental la entrega del ngloon (consejo) mediante distintas formas como son los epew (cuentos), pewmas (sueños) y fenyantun (leyendas).

En este sentido, el Ngülam es la reproducción y socialización de las normas bajo las cuales se formará el actuar de los hijos. Esta transmisión de saberes involucra recordar el pasado, por lo que es necesario desarrollar en los niños la capacidad de saber contar ese pasado y memorizarlo para entregar dichos saberes.

Por último, al reconocer la valoración de la interculturalidad ${ }^{17}$ en Chile, principalmente en la realidad de la región de la Araucanía, esta se cimienta en la

11 Teresita Gallego, "Familias, infancias y crianza: tejiendo humanidad", Revista Virtual Universidad Católica del Norte, n.o 35 (2012).

12 Ibíd.

13 Ziley Mora, Magia y secretos de la mujer mapuche. Sexualidad y sabiduría ancestral (Temuco: Kushé, 1992).

14 Gallego, "Familias, infancias".

15 Ibíd.

16 Daniel Quilaqueo, Segundo Quintriqueo y Prosperino Cárdenas, Educación, currículum e interculturalidad. Elementos sobre formación de profesores en contexto mapuche (Santiago: Frasis editores, 2005).

17 Nelson Aguilera et al., Pueblos Originarios y sociedad nacional en Chile: La interculturalidad en las prácticas sociales, coord. gral. John Durston (Santiago: Programa de las Naciones Unidas para el Desarrollo, 2013). 
construcción de una visión personal de mundo a partir de significados, valores y creencias únicas.

\section{Método}

La metodología utilizada en esta investigación es la cualitativa, es decir, la que "produce datos descriptivos: las propias palabras de las personas, habladas o escritas, y la conducta observable"18. La investigación cualitativa es inductiva: el investigador cualitativo ve a las personas de forma holística, intenta comprenderlas dentro de su propio marco de referencia, en donde todos los escenarios, personas y perspectivas son valiosos y dignos de ser investigados. La investigación cualitativa estudia la realidad en el contexto natural y tal y como sucede, intentando dar sentido o interpretar los fenómenos de acuerdo con los significados que han construido las personas implicadas ${ }^{19}$.

El diseño de esta investigación es el estudio de caso $^{20}$, de tipo instrumental, definido como fenómeno ubicado en tiempo y espacio, que implicó focalizar situaciones abordadas con la profundidad para lograr la comprensión del relato de las abuelas mapuche ${ }^{21}$. El alcance de la investigación corresponde a un estudio descriptivo denso ${ }^{22}$.

Con respecto al rigor, se realizó este proceso de validación de los instrumentos ${ }^{23}$ por el respaldo de personas conocidas en la institución, y adicionalmente en la entrevista se aplicó el rapport lo que facilitó la comunicación con las mujeres mapuche. Se consideró, como una meta de los investigadores, establecer y cultivar relaciones estrechas de confianza para lograr que las personas pudieran manifestar sus vivencias y sentimientos y respondieran libremente el guion de preguntas, generando empatía desde una posición objetiva y confidencial ${ }^{24}$.

Los investigadores asumieron un rol de visitantes ${ }^{25}$, manteniendo una actitud de respeto y coherencia, evitando los prejuicios, mostrándose receptivos con los conocimientos y destrezas, todo lo cual permitió encauzar la entrevista con diligencia, generando en los participantes el sentimiento de aprendizaje. La entrevista se realizó bajo la lógica mapuche de conversación nütram ${ }^{26}$. En este sentido, en una primera aproximación a las comunidades se dio a conocer al

18 Robert Bogdan y Stephen Taylor, Introducción a los métodos cualitativos de investigación. La búsqueda de significados (Barcelona: Paidós Básica, 1987), 20.

19 Juan Mansilla y Claudia Huaiquián, Metodología de la investigación científica. Logos y techné (Temuco: obra independiente, 2015).

20 Robert K. Yin, Case Study Research: Design and Methods (Newbury Park, C.A.: Sage, 1984).

21 Aldo R. Ameigeiras et al., Estrategias de investigación cualitativa, coord. Irene Vasilachis de Gialdino (Barcelona: Gedisa editorial, 2006), 218.

22 Clifford Geertz, La interpretación de las culturas. Capítulo 1: "Descripción densa: hacia una teoría interpretativa de la cultura. (Barcelona: Gedisa editorial, 2003).

23 Uwe Flick, Introducción a la investigación cualitativa (Madrid: Morata, 2007).

24 Mansilla y Huaiquián, Metodología.

25 Ibíd.

26 Juan Mansilla, Claudia Huaiquián y Gabriel Pozo, "Infancia mapuche encerrada. Internados de las escuelas-misiones en la Araucanía, Chile (1900-1935)”, Revista Brasileira de Educação, vol. 23 (2018) http://dx.doi.org/10.1590/S1413-247820182300 
grupo familiar los objetivos y se leyó el consentimiento informado o kimküleal chem zungu mew ñi koneltual ${ }^{27}$. Durante el transcurso de las conversaciones se desarrollaron las siguientes etapas: Chaliwün: saludo que se da en el exterior del hogar familiar, y en el cual se establece un diálogo inicial e invitación a pasar al hogar. Llow witran: es cuando se autoriza el ingreso al hogar en términos de konlepa/konkülepa, wankümu/wanku püle (pasar a tomar asiento). De este modo, es rol de quien llega al hogar iniciar el Pentukun. Yewün: es una forma de evidenciar respeto hacia el hogar visitado, que se puede entender como un "cariño" o "regalo" entregado a la familia. En esta ocasión consistió en yerba mate, azúcar, pan de pueblo y otros alimentos para niños y niñas. Esta es una práctica muy valorada por el pueblo mapuche. Pentukun: la visita pregunta inicialmente por el estado de salud de las personas que integran la familia, y el lof-che, en particular. Después de finalizado el Pentukun sigue una pausa. Wüñoltu pentukun: a la visita se le realizan las mismas preguntas, por la salud, familiares y entorno inmediato. Luego siguieron preguntas con respecto a las identidades de las personas visitantes, sus procedencias geográficas y sanguíneas, etcétera. A partir de este momento la conversación se vuelve más fluida, incluso se integran otros miembros de la familia que no han participado en la primera parte de la interacción. Fill-kake ngütramkan: una vez que la visita se ha dado a conocer y establece una relación amena, se abordan diferentes temas de conversación, que incluyen el estado del ánimo, tiempo climático, estado de las cosechas y aquellas novedades que surjan espontáneamente de la conversación. Esta etapa del diálogo es acompañado del mate, el cual es aceptado con agrado. Entozungun/chalintukun zungu: en esta parte de la conversación se precisan los objetivos del encuentro, la institución u organismo del cual provienen los visitantes, los temas que se conversaran. Este es el momento en que se aplica el consentimiento informado, el cual en este caso es presentado de manera oral, y a la vez, se solicita la autorización para utilizar grabadora de voz. Wiñoltuzungun: una vez reveladas las actividades y objetivos, es decisión de la persona responder inmediatamente a las preguntas o esperar a una siguiente visita para establecer una conversación más fluida. Chalitun: una vez realizadas las propuestas, aclaraciones y hablado sobre temas variados, se procede a la despedida. Esta situación final adquiere importancia y se enfoca nuevamente hacia la persona con la que se llevó a cabo el Pentukun o a la familia en general. Para ello se utilizó la frase wuñopayan may (voy de regreso), agradeciendo la recepción y deseando el bienestar de la familia.

Por consiguiente, se produjo la retirada al campo cuando ya no se encontraron datos, ni nuevos antecedentes, surgiendo así la saturación teórica, que es el criterio para juzgar cuándo dejar de muestrear los diferentes grupos relevantes a una categoría, es decir, que ya no se encuentran datos adicionales que permitan desarrollar las propiedades de una categoría. Una vez determinada la saturación

27 A partir del Proyecto conicyt/fondecyt Regular n. ${ }^{\circ}$ 1160409, titulado "Historia y memoria de la escuela monocultural en la Araucanía (siglos XIX y XX): dispositivos de saber-poder ejercidos por el Estado chileno hacia la sociedad mapuche”. Margarita Canío, Adelaida Canío, Paula Pilquimán y Gabriel Pozo han sistematizado un protocolo cultural de acceso al campo investigativo en contexto mapuche denominado "mapuche ngütramkan", de cuyo texto se han extraído las ideas principales de esta dimensión ética del estudio. 
teórica se realizó la salida del campo, comprometiendo la devolución formal de la información obtenida ${ }^{28}$.

Para complementar se llevó a cabo la triangulación ${ }^{29}$, que se define como una técnica que implica reunir una variedad de datos y métodos para referirlos al mismo tema o problema; implica también que los datos se recojan desde puntos de vista distintos y realizar comparaciones múltiples de un fenómeno único, de un grupo, utilizando perspectivas diversas y múltiples procedimientos.

La triangulación es una técnica o estrategia que permite alcanzar niveles de credibilidad del proceso, con la intención de ampliar el ámbito, densidad y claridad de los constructos desarrollados en el curso de la investigación y corregir los sesgos que aparecen cuando los fenómenos son examinados por un solo observador, con una técnica y desde un solo ángulo de observación ${ }^{30}$.

Mediante la triangulación se procede a recoger todos los antecedentes recabados, permitiendo aumentar la confiabilidad de la investigación, de gran utilidad para los fines de este trabajo, en conjunto con el juicio de experto y la validez teórica ${ }^{31}$.

Los resultados arrojan las siguientes categorías: Internados misionales, evangelización cristiana, control de la salud mapuche, invisibilización del mapuzungun, castigo y disciplinamiento, choque intercultural, pérdida de tierras, censura del mapuzungun, pérdida parcial de la cultura, división social y sexual del trabajo y valores burgueses femeninos occidentales. El contexto de estudio fueron dos comunidades mapuche (lof-che) ubicadas en las comunas de Cholchol y en Maquehue, específicamente. Fueron cinco abuelas mapuche: dos de la comunidad de Maquehue y tres de Cholchol. Se aplicaron entrevistas en profundidad tipo conversación, después se transcribieron las entrevistas, se pocedió a la codificación abierta y axial, para finalmente desarrollar el análisis a partir del muestreo empírico de los documentos primarios generados en campo, y un muestreo teórico, para de este modo realizar los contrastes correspondientes. Las categorías de análisis resultantes fueron las siguientes: (a) presencia de internados misionales, (b) evangelización cristiana, (c) invisibilización del mapunzugun, (d) castigo y disciplinamiento, (e) choque intercultural, (f) pérdida de las tierras, ( $\mathrm{g}$ ) pérdida parcial del mapuzungun, $(\mathrm{h})$ división social y sexual del trabajo, (i) valores burgueses, (j) femeninos occidentales. A continuación se presenta el muestreo empírico desarrollado.

En relación con el código "internados misionales", este se concibe como familia, se debe entender como una tesis intermedia entre las tradiciones pedagógicas del siglo XIX y los postulados de la Escuela Nueva, que resultó instrumental en un mundo de escasos recursos y pérdida de confianza de la escuela mixta ${ }^{32}$. Los

28 Ibíd.

29 Gloria Pérez Serrano, Investigación cualitativa. Retos e interrogantes. II. Técnicas y análisis de datos (Madrid: Editorial La Muralla, 2007).

30 Hugo Cerda, Los elementos de la investigación. Cómo reconocerlos, diseñarlos y construirlos (Quito: abya yala, 1993).

31 Pérez Serrano, Investigación cualitativa.

32 Alicia Civera, "El internado como familia: las escuelas normales rurales en la década de 1920", Revista Latinoamericana de Estudios Educativos, no. 36 (2006); Rafael, Ríos Beltrán y Martha Cerquera Cuéllar. La Modernización de los Contenidos y Métodos de En- 
inicios de los internados en América Latina tuvieron diferentes denominaciones: hogares de menores, hospicios para pobres, asilos, internados parroquiales. Fueron extremadamente modestos y estaban constituidos por salones y piezas equipados con el mobiliario básico y los útiles estrictamente necesarios ${ }^{33}$. De este modo se visualiza en la entrevista: “[...] se quemó, era una escuela con internado, escuela de niñas y la escuela de hombres estaba al otro lado, era una construcción pero grande, como el doble de esta construcción de tres pisos, era con internado" 34 .

En cuanto al código "evangelización cristiana", esta se encuentra desde los primeros instantes en que los colinizadores llegaron a América. Para el caso del pueblo mapuche, esta se llevó a efecto a través de las correrías misioneras de los conquistadores que se instalaron en territorio indígena hasta 1881, situación que se visualizará a través del tiempo en las escuelas-misiones ${ }^{35}$. En este sentido, a la ayuda entregada y a la educación de la mano con la evangelización, "los mapuches entregaron, por ejemplo, más de 100 hectáreas de terrenos a la sociedad misionera anglicana para que construyeran una escuela ${ }^{36}$.

Respecto a la "invisibilización del mapuzungun", este se visualiza en las siguientes textualidades:

Fue un terrible error de mis padres y de todos los profesores que no nos dejaron practicar el mapuzungun si yo después de vieja puedo decir de adulta eh, pude aprender o entender el mapuzungun, eso no se nos debiera haber quitado y eso se quitaba para qué, decían que si nosotros ya estábamos en esta cultura de castellano que se nos iba a enredar la lengua, que no íbamos saber hablar y que nos iban a discriminar ${ }^{37}$.

Del mismo se reconoce lo siguiente:

Sí, pero si mi padre decía que la llegada de quizá de los españoles traían adelantos en la educación, en la ciencia, entonces no por eso él quería que nosotros nos insertáramos y saliéramos un poco no de la cultura, si no que de, cómo le dijera, de campo a tener otra vida más digna según ${ }^{38}$.

En este orden de cosas, la invisibilización de los saberes mapuche se manifiesta especialmente en el bloqueo del uso de la lengua materna, pues el idioma con el cual se enseñaron los contenidos en sectores con una alta densidad de

señanza: Reflexiones sobre la Escuela Nueva en Colombia. Revista Historia de la Educación Latinoamericana vol.16 no. 22 (2014): 157-72. https://doi.org/10.19053/01227238.2693.

33 José Muriá y Jaime Olveda, Educación y cultura. Lecturas históricas de Guadalajara (D. F.: Instituto Nacional de Antropología e Historia, 1992).

34 Entrevistada 1. Entrevista realizada en la localidad de Maquehue.

35 Mansilla, Huaiquián y Pozo, "Infancia mapuche”.

36 María Riveros, "Religión e identidad en el pueblo mapuche", trabajo presentado en el seminario "Problemas de la cultura latinoamericana" (Santiago: Universidad de Chile, 2000).

37 Entrevistada 4. Entrevista realizada en la localidad de Cholchol.

38 Entrevistada 4. Entrevista realizada en la localidad de Cholchol. 
población indígena fue el castellano ${ }^{39}$, donde quedaba prohibido hablar el mapuzugun: todo debía ser respondido en español.

En relación con el código "castigo y disciplinamiento", se viualiza la siguiente textualidad extraída de las entrevistas:

[...] y había que hacer la tarea porque, si no, ahí sí que había castigo sin tarea... el profesor usaba una regla, un reglazo aquí en la palma de su mano el que no tenía la tarea, entonces había que llevar la tarea ${ }^{40}$. [...] bueno, en ese entonces la educación era más rígida y podían dar un tablazo, podían dar un varillazo a quien hiciera algo indebido, supongamos como, no sé, sacarle las cosas a otro compañero o simplemente salirse de las reglas que tenían establecidas el internado digamos entonces ${ }^{41}$. [...] y ahí veíamos cómo les pegaban a las niñas internas; una vez encontramos a dos niñas amarradas con el pelo largo, igual como dos animalitos castigadas las tenían, eso lo hacían las monjitas porque ellas estaban a cargo de las niñas ${ }^{42}$.

Por otra parte tenemos el código "choque intercultural":

[...] eh, yo creo que quizás al inicio haya sido como, eh, chocante para algunas familias, pero yo creo que al ver que llegó una gran cantidad tratando de trabajar para la educación, para evangelizar y para ayudar en la salud; yo creo que no fue tan notoria esa división o ese encontrón, que podríamos decir, entre las dos culturas ${ }^{43}$.

Otro código muy relevante corresponde a la "pérdida de tierras", el cual se se refleja en la siguiente cita empírica:

[...] seguramente ellos visitaron y dieron a conocer sus planteamientos, sus planes que tenían, y como que convencieron fácilmente, porque con las facilidades que se les entregó una tremenda cantidad de terreno, porque la gente quería civilizarse o quizás como se las arreglaron para poder lograr que los mapuches entreguen terreno para la educación $n^{44}$.

A su vez, el código "pérdida parcial de la cultura" se visualiza en la siguiente textualidad:

[...] pero sí, una vez más voy a manifestar que es una pena tremenda que hayamos perdido, más bien, hayamos perdido parte de nuestra cultura, de nuestras costumbres, nuestras tradiciones, y eso es por, quizás, por malas orientaciones, malas orientaciones a nuestra gente, a nuestros antepasados, porque nuestra cul-

\footnotetext{
39 Juan Mansilla, Daniel Llancavil, Manuel Mieres y Elizabeth Montanares, "Instalación de la escuela monocultural en la Araucanía, 1883-1910: dispositivos de poder y Sociedad Mapuche”, Educação e Pesquisa, n.o 1 (2016).

40 Entrevistada 5. Entrevista realizada en la localidad de Cholchol.

41 Entrevistada 5. Entrevista realizada en la localidad de Cholchol.

42 Entrevistada 5. Entrevista realizada en la localidad de Cholchol.

43 Entrevistada 2. Entrevista realizada en la localidad de Maquehue.

44 Entrevistada 2. Entrevista realizada en la localidad de Maquehue.
} 
tura, nuestras tradiciones, no era, eh, para olvidarla por el tema de incorporarnos, insertarnos a la cultura que tenemos ${ }^{45}$. forma:

El código "división social y sexual del trabajo", se expresa de la siguiente

[...] más que nada la forma de vestir por los coloríos que uno usa siempre, más en el campo, que uno es más... llegue con un vestido colorido y era como que desentonaba con el resto, claro, se notaba la diferencia de quienes éramos del campo, digamos entre comillas, también tanto mapuche como no mapuches, ya el hecho de ser del campo ya éramos diferentes pero uno se fue adaptando y de hecho, igual la vestimenta, claro, y cuando venía mi mamá a verme, vino como en primer año, creo, sí, que ella se vestía de mapuche, entonces también era que llamaba la atención acá en... bueno, que en ese entonces venían varias apoderadas y entonces no era como tan, tan llamativo, pero si algo [...] las asignaturas que habían o los talleres que tenía el colegio, porque también habían muchas mujeres... hacían como talleres especiales para mujeres, otros para hombres o era una cosa más general para todos... habian distinciones en la enseñanza dirigida hacia mujeres, dirigidas hacia hombres, como enseñándoles manualidades ${ }^{46}$.

Finalmente, el código "valores burgueses femeninos occidentales" se visualiza en la siguiente textualidad:

Que las mujeres, bueno, de hecho, bíblicamente nos decían de que Dios creó a los hombres y las mujeres... hay dos sexos, por lo tanto las mujeres en esto se definen, sea ser femenina es así señorita [...] de hecho usábamos el jumper a cierta medida, era, como todo, peinaditas, era como todo eso,... estaban todas esas herramientas para poder aprender, de ser mujer, eso siempre no dictaban en los consejos... el jumper, ese jumper negro azul marino, así es que ahí abajito de las rodillas, en ese entonces era como bien marcada la... el feminismo y lo que es un hombre y una mujer. No había esa libertad de como a ser por ejemplo en el campo si tu abuela hacía todo eso no tenía a lo mejor eso de ser mujer como muy marcado por que también hacía cosas de criar animales por que podían ser actividades de hombre ${ }^{47}$

\section{CONCLUSIÓN}

Los internados fueron la estructura organizacional imprescindible para lograr los objetivos de adoctrinamiento disciplinario y control social pensados desde la escuela y misión. Las abuelas expresan en sus relatos que cuando eran estudiantes, al permanecer internas, disminuyeron sus posibilidades de ausentismo y deserción escolar, situación que se presentaba con más frecuencia en

45 Entrevistada 5. Entrevista realizada en la localidad de Cholchol.

46 Entrevistada 3. Entrevista realizada en la localidad de Maquehue.

47 Entrevistada 4. Entrevista realizada en la localidad de Cholchol. 
las temporadas agrícolas y ceremonias religiosas como el guillatue. Los internados también se transformaron en un alivio para la economía doméstica de las familias mapuche porque allí podían comer todos los días. Se hace necesaria una revisión que involucre a otros actores educativos: los niños y las niñas. Las diferencias formativas de género aparecen como una dimensión de gran interés para comprender la ideología curricular que se operacionalizó en los contenidos pedagógicos que se enseñaron en escuelas y liceos de la Araucanía. La escuela fiscal, así como la instrumentalización estatal de las escuelas misionales católicas, en específico, las escuelas misionales levantadas por la congregación de los capuchinos bávaros a partir de la década de 1890, fracasaron desde su origen en su contribución para el encuentro entre los grupos que compartieron el despojado territorio mapuche. La escuela monocultural ha cumplido un papel central en la función de chilenizar a la población indígena a través de la transmisión de conocimiento, así como valores, normas y rituales colectivos, además de la difusión de símbolos y sentido de pertenencia a una comunidad diferente. En esta línea, se llevó (y se lleva) a cabo un proceso de intervención que pretende uniformar, pero no consigue integrar del todo. La educación y la religión fueron las herramientas que facilitaron la inserción social del mapuche sobreviviente, de acuerdo con un modelo homogeneizador.

\section{REFERENCIAS}

Aguilera, Nelson, Ana Ancapi, Lenina Barrios, Álvaro Bello, Natalia Caniguan et al. Pueblos Originarios y sociedad nacional en Chile: La interculturalidad en las prácticas sociales. Coordinador general John Durston. Santiago: Programa de las Naciones Unidas para el Desarrollo, 2012.

Ameigeiras, Aldo R., Lilia B. Chernobilsky, Verónica Giménez, Fortunato Mallimaci, Nora Mendizábal et al. Estrategias de investigación cualitativa. Coordinado por Irene Vasilachis de Giordino. Madrid: Gedisa editorial, 2006.

Bogdan, Robert y Stephen Taylor. Introducción a los métodos cualitativos de investigación. La búsqueda de significados. Barcelona: Paidós Básica, 1987.

Caro, Aracely y Julio Terencán. "El Ngülam en el discurso intrafamiliar mapuche”. Iberóforum. Revista de Ciencias Sociales de la Universidad Iberoamericana I, n. ${ }^{\circ} 1$ (2006): 1-9.

Cerda, Hugo. Los elementos de la investigación. Cómo reconocerlos, diseñarlos y construirlos. Quito: ABYA YALA, 1993.

Corporación Nacional de Desarrollo Indígena (CONADI). Ley indígena. Ley n. ${ }^{\circ} 19.253$ d. of. 5. $.^{\circ} 10 .{ }^{\circ}$ 1993. Temuco: conAdi. http://www.leychile.cl/Navegar?idNorma=30620

Civera, Alicia. "El internado como familia: las escuelas normales rurales en la década de 1920". Revista Latinoamericana de Estudios Educativos 36, n. ${ }^{\circ}$ 3-4 (2006): 53-73.

Eagleton, Terry. La idea de cultura. Una mirada politica sobre los conflictos culturales. Barcelona: Paidós, 2001.

Flick, Uwe. Introducción a la investigación cualitativa. Madrid: Morata, 2007.

Gallego, Teresita. "Familias, infancias y crianza: tejiendo humanidad". Revista Virtual Universidad Católica del Norte, n. ${ }^{\circ} 35$ (2012): 63-82.

Geertz, Clifford. La interpretación de las culturas. Barcelona: Gedisa editorial, 2003.

Gleisner, Christine y Sara Montt. Mapuche. Serie introducción histórica y relatos de los pueblos originarios de Chile. Santiago: FUCOA-Fondart, 2014.

INE (Instituto Nacional de Estadísticas de Chile). “Censo 2012, síntesis de resultados". Santiago: INE, 2012. 
Mansilla, Juan y Claudia Huaiquián. Metodología de la investigación científica. Logos y techné. Temuco: obra independiente, 2015.

Mansilla, Juan, Daniel Llancavil, Manuel Mieres y Elizabeth Montanares. "Instalación de la escuela monocultural en la Araucanía, 1883-1910: dispositivos de poder y Sociedad Mapuche". Educação e Pesquisa, n. ${ }^{\circ} 1$ (2016): 213-228.

Mansilla Sepúlveda, Juan, Claudia Huaiquián Billeke y Gabriel Pozo Menares. “Infancia mapuche encerrada: internados de las escuelas-misiones en la Araucanía, Chile (1900-1935)". Revista Brasileira de Educação 23 (2018): 2-28.

Mora, Ziley. Magia y secretos de la mujer mapuche. Sexualidad y sabiduría ancestral. Temuco: Kushé, 1992.

Muriá, José y Jaime Olveda. Educación y cultura. Lecturas históricas de Guadalajara. D. F.: Instituto Nacional de Antropología e Historia, 1992.

Pérez Serrano, Gloria. Investigación cualitativa. Retos e interrogantes. II. Técnicas y análisis de datos. Madrid: Editorial La Muralla, 2011.

Quilaqueo, Daniel, Segundo Quintriqueo y Prosperino Cárdenas. Educación, currículum e interculturalidad. Elementos sobre formación de profesores en contexto mapuche. Santiago: Frasis editores, 2005.

Ríos Beltrán Rafael y Cerquera Cuéllar, Martha. La Modernización de los Contenidos y Métodos de Enseñanza: Reflexiones sobre la Escuela Nueva en Colombia. Revista Historia de la Educación Latinoamericana vol.16 no. 22 (2014): 157-72. https://doi.org/10.19053/01227238.2693.

Riveros, María. Religión e identidad en el pueblo Mapuche. Trabajo presentado en el seminario “Problemas de la cultura latinoamericana". Santiago: Universidad de Chile, 2000.

Sadler, Michelle y Alexandra Obach. Pautas de crianza mapuche. Estudio "Significaciones, actitudes y prácticas de familias mapuches en relación a la crianza y cuidado infantil de los niños y niñas desde la gestación hasta los cinco años". Santiago: Ministerio de Planificación, 2006.

Yin, Robert K. Case Study Research: Design and Methods. Newbury Park, C.A.: Sage, 1984.

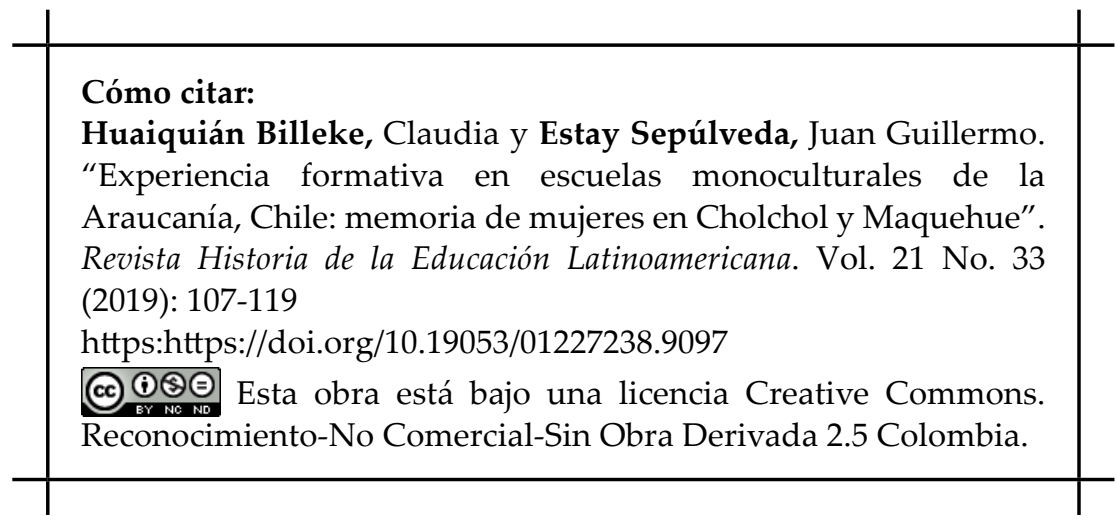

